\title{
PHOTOPRODUCTION OF THE $\Lambda(1405)$ ON THE PROTON AND NUCLEI
}

\author{
J.C. Nacher ${ }^{1,2}$, E. Oset ${ }^{1,2}$, H. Toki ${ }^{1}$ and A. Ramos ${ }^{3}$ \\ ${ }^{1}$ Research Center for Nuclear Physics (RCNP), Osaka University, Ibaraki, \\ Osaka 567-0047, Japan. \\ ${ }^{2}$ Departamento de Física Teórica and IFIC, Centro Mixto Universidad de \\ Valencia-CSIC 46100 Burjassot (Valencia), Spain. \\ ${ }^{3}$ Departament d'Estructura i Constituents de la Materia, Universitat de \\ Barcelona, Diagonal 647, 08028 Barcelona, Spain.
}

\begin{abstract}
We study the $\gamma p \rightarrow K^{+} \Lambda(1405)$ reaction at energies close to threshold using a chiral unitary model where the resonance is generated dynamically from $K^{-} p$ interaction with other channels constructed from the octets of baryons and mesons. Predictions are made for cross sections into several channels and it is shown that the detection of the $K^{+}$is sufficient to determine the shape and strength of the $\Lambda(1405)$ resonance. The determination of the resonance properties in nuclei requires instead the detection of the resonance decay channels. Pauli blocking effects on the resonance, which have been shown to be very important for the resonance at rest in the nucleus, are irrelevant here where the resonance is produced with a large momentum. The nuclear modifications here would thus offer information on the resonance and $K^{-}$nucleus dynamics complementary to the one offered so far by $K^{-}$ atoms.
\end{abstract}


The $S_{01} \Lambda(1405), \mathrm{I}\left(J^{P}\right)=0\left(\frac{1}{2}^{-}\right)$, resonance is situated just below the $K^{-} N$ threshold and plays an important role in the $\bar{K} N$ interaction at low energies [1]. The presence of this resonance has an important role in the properties of kaons in nuclear matter and nuclei. Modifications of the resonance properties in nuclei lead to substantial changes in the kaon selfenergy in the medium and have repercussions in atoms which have been often emphasized $[2,3]$.

The $\Lambda(1405)$ resonance is well described within a unitary coupled channel approach, as shown earlier in $[4,5]$ and more recently in $[6,7,8,9]$. An important step in the understanding of the $\Lambda(1405)$ dynamics has been done in $[7,8,9]$ where, using chiral Lagrangians and a unitary coupled channel approach, the properties of the resonance, as well as the low energy $K^{-} N$ cross sections, are well reproduced. The use of nonperturbative unitary approaches in coupled channels shows here its strength over ordinary perturbation schemes which would be unable to generate a resonance structure in the scattering matrix.

In ref. [9], where the whole set of mesons of the pseudoscalar octet, as well as those of the octet of stable baryons, are used, one can reproduce all the low energy $K^{-} p$ data, as well as the properties of the $\Lambda(1405)$ resonance by means of the lowest order chiral Lagrangian with only one parameter, a suitable cut off. This cut off is needed to regularize the loop integrals in the Bethe Salpeter equation and, at the same time, allows to effectively incorporate effects of the higher order chiral Lagrangians. These findings, similar to those in $[7,8]$, favour the interpretation of the latter resonance as a quasibound state of mesons and baryons, different in nature to genuine baryons of $3 q$ internal structure which would survive in the large $N_{C}$ limit [10].

One of the interesting findings in the nuclear modifications of the $\Lambda(1405)$ resonance is the effect of Pauli blocking in the intermediate states of the coupled channels, which moves the resonance to higher energies and changes the sign of the $K^{-} p$ amplitude at threshold from repulsive to attractive [11, 12]. The attraction in the medium is suggested by the analysis of the the data of kaonic atoms $[2,3,13,14]$. This attraction in the medium could eventually lower the kaon mass sufficiently to produce kaon condensates in a neutron star [15]. However, a recent selfconsistent determination of the $K^{-}$selfenergy in the medium, accounting for Pauli blocking and the effect of the kaon selfenergy itself, leads to substantially different results in the sense that the position of the resonance barely moves in the medium [16]. It becomes wider while some attraction on the kaons is also generated. A more recent calculation, incorporating in addition the selfenergy of the pions in the intermediate $\Sigma \pi$ and $\Lambda \pi$ channels, comes to support these latter findings about the position, only the width becomes larger and at densities close to nuclear matter the resonance structure disappears [17]. A net attraction on the $K^{-}$is generated in this approach at densities higher than $\rho_{0} / 10$.

As one can see, the theoretical situation is at a stage where experimental information is needed to settle the questions, which are also essential to put the hypothesis of the kaon condensation on firmer grounds. The present work 
suggests a nuclear reaction where an answer to some of these questions can be obtained. The reaction is photoproduction of the $\Lambda(1405)$ resonance on the proton and in nuclei, which can be easily implemented in present facilities like TJNAF and LEPS of SPring8/RCNP. The elementary reaction is

$$
\gamma p \rightarrow K^{+} \Lambda(1405) \text {. }
$$

In order to get the coupling of the mesons and the photon to the $\Lambda(1405)$ resonance we make use of the model of [9] for meson-baryon interaction which generates the resonance dynamically from the 10 coupled channels, $K^{-} p, \bar{K}^{0} n$, $\pi^{0} \Lambda, \pi^{0} \Sigma^{0}, \eta \Lambda, \eta \Sigma^{0}, \pi^{+} \Sigma^{-}, \pi^{-} \Sigma^{+}, K^{+} \Xi^{-}, K^{0} \Xi^{0}$. The starting point is the meson-baryon amplitude originated from the lowest order chiral Lagrangian $[18,19,20]$ which generates the coupling for the $M_{i} B_{i} \rightarrow M_{j} B_{j}$ transition [9]

$$
V_{i j}=-C_{i j} \frac{1}{4 f^{2}} \bar{u}\left(p^{\prime}\right) \gamma^{\mu} u(p)\left(k_{\mu}+k_{\mu}^{\prime}\right),
$$

where $f$ is the decay constant of the pion $\left(f_{\pi}=93 \mathrm{MeV}\right)$. In [9] this constant is taken as an average between the one for pions and kaons, $f=1.15 f_{\pi}$. In eq. (2) $k_{\mu}$ and $k_{\mu}^{\prime}$ are the initial and final meson momenta and $C_{i j}$ is a $10 \times 10$ matrix which is shown in table 1 of [9]. For the low energies which we will consider here only the s-wave is relevant and the expression of eq. (2) is further simplified to

$$
V_{i j}=-C_{i j} \frac{1}{4 f^{2}}\left(k^{0}+k^{\prime 0}\right) .
$$

The scattering matrix between two channels, $T_{i j}$, is then given by means of the Bethe Salpeter equation, where $\mathrm{V}$ and $\mathrm{T}$ are shown to factorize on shell outside the loop integral using the heavy baryon approximation [9]. Hence one has the algebraic matrix equation

$$
T=V+V G T \rightarrow T=[1-V G]^{-1} V
$$

with $\mathrm{G}$ a diagonal matrix, with matrix elements

$$
G_{l}=i \int \frac{d^{4} q}{(2 \pi)^{4}} \frac{M_{l}}{E_{l}(\vec{q})} \frac{1}{k^{0}+p^{0}-q^{0}-E_{l}(\vec{q})+i \epsilon} \frac{1}{q^{2}-m_{l}^{2}+i \epsilon}
$$

corresponding to a loop diagram with an intermediate meson and baryon propagators and which is regularized with a cut off in $\vec{q}$, with $q_{\max }=630 \mathrm{MeV}$ in [9], the only parameter fitted to the data.

The $\Lambda(1405)$ is a s-wave resonance and thus we concentrate on mechanisms which allow s-wave baryon-meson production. In a first step the reaction would proceed as depicted diagrammatically in Fig. 1 which forms a gauge invariant set of diagrams at threshold of the pair meson production. In order to simplify the theoretical calculations we choose a photon energy around $E_{\gamma L a b}=1.7 \mathrm{GeV}$ where the particles are produced with low energy. In that case it is easy to prove that the contribution of the meson pole terms (diagrams a, b, of Fig. 
1 ) is negligible (less than $5 \%$ contribution) and one can live with the contact term alone (diagram c). This choice of energy is also suited theoretically to prevent the contribution of terms like in Fig. 1a, where the photon couples directly to the nucleon (or excites resonances) and then one has the contact vertex MMBB. In such a case one gets the combination $\left(k_{0}^{\prime}-k_{0}\right)$ in eq. (3) which is a very small number. Similarly, other terms where the mesons would be produced at different vertices would involve p-wave couplings which would also vanish at threshold. The smallness of the momenta of the mesons involved is also suited to prevent the formation of a p-wave resonance like the $\Sigma(1385)$. In any case the small branching ratio $(12 \%)$ of this resonance into the $\pi \Sigma$ channel makes easy the separation from the $\Lambda(1405)$ which decays only into that channel.

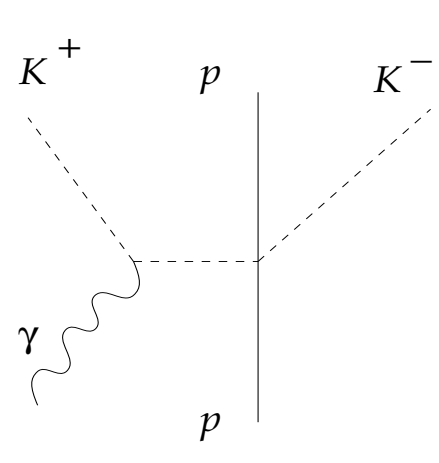

a)

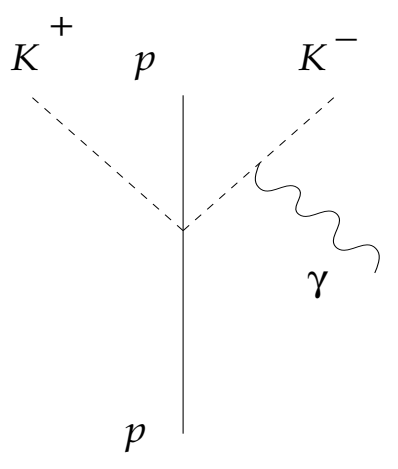

b)

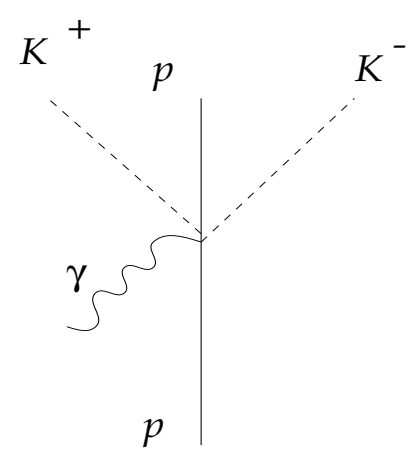

c)

Figure 1: Feynman diagrams used in the model for the $\gamma p \rightarrow K^{+} \Lambda(1405)$ reaction.

Minimal coupling from eq. (2) provides the contact term $\gamma M_{i} B_{i} \rightarrow M_{j} B_{j}$

$$
V_{i j}^{(\gamma)}=C_{i j} \frac{e}{4 f^{2}}\left(Q_{i}+Q_{j}\right) \bar{u}\left(p^{\prime}\right) \gamma^{\mu} u(p) \epsilon_{\mu}
$$

where $Q_{i}, Q_{j}$ are the initial and final meson charges and $\epsilon_{\mu}$ the photon polarization vector.

In this situation close to threshold which we have chosen we can neglect terms proportional to the final baryon momentum, which are small in the kinematics chosen. In this case the contact term of Fig. 1c for $\gamma p \rightarrow K^{+} M_{j} B_{j}$ (or equivalently $\gamma p K^{-} \rightarrow M_{j} B_{j}$ ), where $M_{j} B_{j}$ is any of the 10 states that couple to the $\Lambda(1405)$, can be written in the Coulomb gauge $\epsilon^{0}=0, \vec{\epsilon} \cdot \vec{q}=0$ and in the $\gamma p$ CM frame as

$$
V_{j}^{(\gamma)}=C_{1 j} \frac{e}{4 f^{2}} \frac{i(\vec{\sigma} \times \vec{q}) \vec{\epsilon}}{2 M} Q_{j}^{\prime}
$$


where the index 1 in $C_{1 j}$ stands for the $K^{-} p$ channel and $Q_{j}^{\prime}=1-Q_{j}$. Here $Q_{j}^{\prime}$ is the vector $(2,1,1,1,1,1,0,2,0,1)$ corresponding to the final states in the order mentioned in the introduction and $\mathrm{M}$ stands for the proton mass.

The $\Lambda(1405)$ resonance is generated dynamically by iteration of the $\mathrm{MB} \rightarrow$ M'B' vertex according to the Bethe Salpeter equation, and diagrammatically this is shown in Fig. 2.
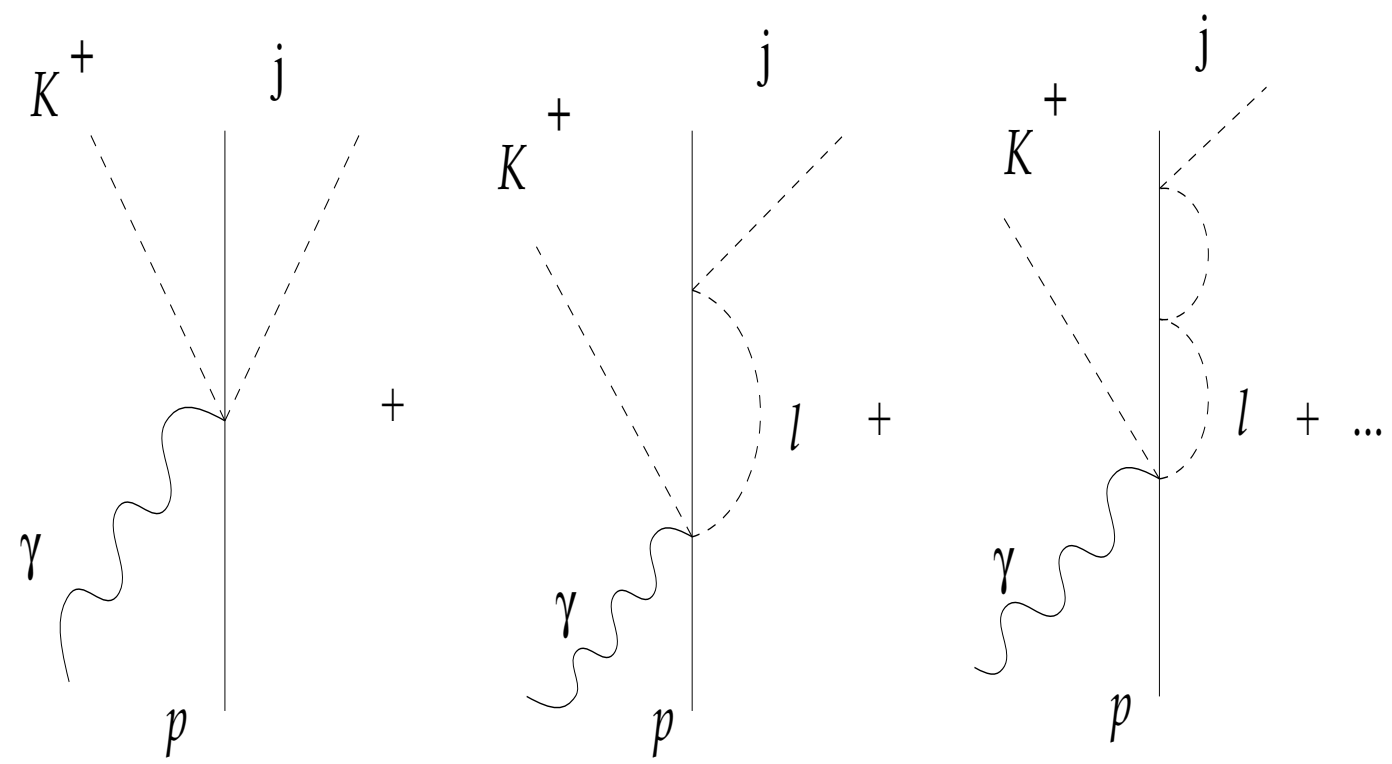

Figure 2: Diagrammatic representation of the meson-baryon final state interaction in the $\gamma p \rightarrow K^{+} \Lambda(1405)$ process.

The sum implicit in Fig. 2 is easily evaluated. The $t_{j}^{(\gamma)}$ matrix for the process with the $\mathrm{j}$ channel in the final state is given by means of the vector $D_{j}=C_{1 j} \cdot Q_{j}^{\prime}$ as:

$$
t_{j}^{(\gamma)}=\frac{e}{4 f^{2}} \frac{i(\vec{\sigma} \times \vec{q}) \vec{\epsilon}}{2 M}\left(D_{j}+\sum_{l} D_{l} G_{l} T_{l j}\right),
$$

where the on shell factorization of the strong amplitude found in [9] and in [21] for the related electromagnetic process has been used. The particular structure of eq. (8) allows one to obtain an easy formula for the invariant mass distribution of the final $\mathrm{j}$ state, particularly suited to the search of a resonance. We find

$\left.\frac{d \sigma}{d M_{I}}\right|_{j}=\frac{1}{(2 \pi)^{3}} \frac{1}{4 s} \frac{M M_{j}}{s-M^{2}} \frac{1}{M_{I}} \sum \sum\left|t_{j}^{(\gamma)}\right|^{2} \lambda^{1 / 2}\left(s, M_{I}^{2}, m_{K}^{2}\right) \lambda^{1 / 2}\left(M_{I}^{2}, M_{j}^{2}, m_{j}^{2}\right)(9)$

where $M_{I}$ is the invariant mass of the $\mathrm{j}$ state, $M_{j}, m_{j}$ the masses of the $\mathrm{j}$ state and $\lambda(\mathrm{x}, \mathrm{y}, \mathrm{z})$ the ordinary Källen function.

In Fig. 3 we show $\frac{d \sigma}{d M_{I}}$ for the different channels. While all coupled channels collaborate to the built up of the $\Lambda(1405)$ resonance, most of them open up at higher energies and the resonance shape is only visible in the $\pi^{+} \Sigma^{-}, \pi^{-} \Sigma^{+}$, 
$\pi^{0} \Sigma^{0}$ channels. The $\bar{K} N$ production occurs at energies slightly above the resonace and the $\pi^{0} \Lambda$, with isospin one, only provides a small background below the resonance.

It is interesting to see the different shapes of the three $\pi \Sigma$ channels. This can be understood in terms of the isospin decomposition of the states

$$
\begin{aligned}
& \left|\pi^{+} \Sigma^{-}\right\rangle=-\frac{1}{\sqrt{6}}|2,0\rangle-\frac{1}{\sqrt{2}}|1,0\rangle-\frac{1}{\sqrt{3}}|0,0\rangle \\
& \left|\pi^{-} \Sigma^{+}\right\rangle=-\frac{1}{\sqrt{6}}|2,0\rangle+\frac{1}{\sqrt{2}}|1,0\rangle-\frac{1}{\sqrt{3}}|0,0\rangle \\
& \left|\pi^{0} \Sigma^{0}\right\rangle=\sqrt{\frac{2}{3}}|2,0\rangle-\frac{1}{\sqrt{3}}|0,0\rangle
\end{aligned}
$$

Disregarding the $\mathrm{I}=2$ contribution which is negligible, the cross sections for the three channels go as:

$$
\begin{array}{ll}
\frac{1}{2}\left|T^{(1)}\right|^{2}+\frac{1}{3}\left|T^{(0)}\right|^{2}+\frac{2}{\sqrt{6}} \operatorname{Re}\left(T^{(0)} T^{(1) *}\right) ; & \pi^{+} \Sigma^{-} \\
\frac{1}{2}\left|T^{(1)}\right|^{2}+\frac{1}{3}\left|T^{(0)}\right|^{2}-\frac{2}{\sqrt{6}} \operatorname{Re}\left(T^{(0)} T^{(1) *}\right) ; & \pi^{-} \Sigma^{+} \\
\frac{1}{3}\left|T^{(0)}\right|^{2} ; \quad \pi^{0} \Sigma^{0} &
\end{array}
$$

The crossed term $T^{(0)} T^{(1) *}$ is what makes these cross sections different. We can also see that

$$
\begin{aligned}
& 3 \frac{d \sigma}{d M_{I}}\left(\pi^{0} \Sigma^{0}\right) \simeq \frac{d \sigma}{d M_{I}}(I=0) \\
& \frac{d \sigma}{d M_{I}}\left(\pi^{0} \Sigma^{0}\right)+\frac{d \sigma}{d M_{I}}\left(\pi^{+} \Sigma^{-}\right)+\frac{d \sigma}{d M_{I}}\left(\pi^{-} \Sigma^{+}\right) \simeq \frac{d \sigma}{d M_{I}}(I=0)+\frac{d \sigma}{d M_{I}}(I=1)(16
\end{aligned}
$$

This means that the real shape of the resonance must be seen in either the $\pi^{0} \Sigma^{0}$ channel or in the sum of the three $\pi \Sigma$ channels, provided the $\mathrm{I}=1$ cross section (not the crossed terms which are relatively large ) is small as it is the case. Incidentally, eqs. $(13,14)$ also show that the difference between the $\pi^{+} \Sigma^{-}$and $\pi^{-} \Sigma^{+}$cross sections gives the crossed term and hence provides some information on the $\mathrm{I}=1$ amplitude.

In Fig. 4 we recombine the results in a practical way from the experimental point of view. We show the $\mathrm{I}=0$ contribution, the $\Sigma^{0} \pi^{0}$ contribution and the sum of all channels including the $\pi^{0} \Lambda$, and we see that they are all very similar and the total contribution is just the $\Lambda(1405)$ contribution plus a small background. In practical terms this result means that the detection of the $K^{+}$ 
alone (which sums the contribution of all channels) is sufficient to determine the shape and the strength of the $\Lambda(1405)$ resonance in this reaction.

The study of the reaction in nuclei requires special care. Indeed, assume one uses the same set up as before with a nuclear target and measures the outgoing $K^{+}$. There the invariant mass will be given by

$$
\begin{aligned}
M_{I}^{2}(p)=(q+p-k)^{2}= & M^{2}+m_{K}^{2}-2 q^{0} k^{0}+2 \vec{q} \cdot \vec{k}+ \\
& 2 p^{0}\left(q^{0}-k^{0}\right)-2 \vec{p} \cdot(\vec{q}-\vec{k})
\end{aligned}
$$

with $q, k, p$ the momenta of the photon, $K^{+}$and initial proton respectively. Since $\vec{q}-\vec{k}$ has a large size, there will be a large spreading of invariant masses due to Fermi motion for a given set up of photon and $K^{+}$momenta, unlike in the free proton case where $M_{I}^{2}$ is well determined $[$. The nuclear cross section normalized to the number of protons (the neutrons through $K^{-} n$ and coupled channels only contribute to $\mathrm{I}=1$ with a small background) would be given by the convolution formula

$$
\left.\frac{1}{Z} \frac{d \sigma}{d M_{I}}\right|_{A} \simeq \frac{2}{\rho_{p}} \int \frac{d^{3} p}{(2 \pi)^{3}} \frac{d \sigma}{d M_{I}(\vec{p})} \quad ; \quad \rho_{p}=\frac{k_{F}{ }^{3}}{3 \pi^{2}}
$$

where the integral over $\vec{p}$ ranges up to the Fermi momentum $k_{F}$.

In order to show the effects of the Fermi motion we choose values of $\vec{k}$ corresponding to forward $K^{+}$in the CM (and hence largest value of $\vec{k}$ in the lab frame) which would minimize the spreading of the $M_{I}^{2}(\vec{p})$ in eq. (17). Even then, we can see in Fig. 4 the result of the distribution. The $M_{I}$ in the $\mathrm{x}$ axis of the figure in this case is taken for reference from eq. (17) for a nucleon at rest. We can see that the spreading of the invariant masses is so large that one looses any trace of the original resonance. This simply means that in order to see genuine dynamical effects one would have to look at the invariant mass of the resonance from its decay product, $\pi \Sigma$, tracing back this original invariant mass with appropiate final state interaction corrections.

One interesting thing here is that the $\Lambda(1405)$ resonance is produced with a large momentum in the nuclear lab frame. We have checked that Pauli blocking effects in the resonance, which were so important for the resonance at rest in the nucleus, become now irrelevant. Hence medium modifications of the resonance in the present situation should be attributed to other dynamical effects [17].

The flattering of the resonance in nuclei when only the $K^{+}$is detected has its positive aspects though. Indeed by using $\mathrm{CH}_{2}$ targets instead of just $H$, as planned in SPring8/RCNP, and detecting only the $K^{+}$, one would be observing the clear signal of the $H$ on top of a flat background of the ${ }^{12} \mathrm{C}$.

The cross sections obtained, of the order of $5 \mu \mathrm{b} / \mathrm{GeV}$, are in the easily attainable range in present experimental facilities like TJNAF and LEPS of SPring8/RCNP. The results obtained in this work should encourage the performance of the actual experiments. They would serve to test current ideas

\footnotetext{
${ }^{1}$ We are endebted to T. Nakano and J. K. Ahn for calling us the attention on this point
} 
on chiral symmetry from the elementary reaction and with nuclear targets would give us much needed information on the in medium properties of the $\Lambda$ (1405) resonance and $K^{-}$. This should help resolve questions like the $K^{-}$ condensation, and the origin of the attraction seen in $K^{-}$atoms.

Acknowledgements. We acknowledge useful discussions and comments from A. Titov, T. Nakano and J. K. Ahn. We are grateful to the COE Professorship program of Monbusho, which enabled E. O. to stay at RCNP to perform the present work. One of us, J.C. Nacher would like to acknowledge the hospitality of the RCNP of the Osaka University where this work was done and support from the Ministerio de Educacion y Cultura. This work is partly supported by DGICYT contract number PB96-0753 and PB95-1249.

\section{References}

[1] A. D. Martin, Nucl. Phys. B179 (1981) 33

[2] R. Brockmann, W. Weise and L. Tauscher, Nucl. Phys. A308 (1978) 365

[3] M. Mizoguchi, S. Hirenzaki and H. Toki, Nucl. Phys. A567 (1994) 893

[4] R. H. Dalitz and S. F. Tuan, Ann. Phys. (N.Y.) 10 (1960) 307; R.H. Dalitz, T. C. Wong and G. Rajasekaran, Phys. Rev. 153 (1967) 1617

[5] A. D. Martin, Nucl. Phys. B179 (1981) 33

[6] P.B. Siegel and B. Saghai, Phys. Rev. C52 (1995) 392

[7] N. Kaiser, R. Siegel and W. Weise, Nucl. Phys. A594 (1995) 325

[8] N. Kaiser, T. Waas and W. Weise, Nucl. Phys. A612 (1997) 297

[9] E. Oset and A. Ramos, Nucl. Phys. A635 (1998) 99

[10] G. Ecker, J. Gasser, H. Leutwyler, A. Pich and E. de Rafael, Phys. Lett. B223 (1989) 425

[11] V. Koch, Phys. Lett. B337 (1994) 7

[12] T. Waas, N. Kaiser and W. Weise, Phys. Lett B365 (1996) 12

[13] C. J. Batty, Nucl. Phys. A372 (1981 ) 418

[14] C. J. Batty, E. Friedman and A. Gal, Phys. Rep. 287 (1997) 385 
[15] G. E. Brown, K. Kubodera, M. Rho and V. Thorsson, Phys. Lett. B291 (1992) 355; C.H. Lee, G.E. Brown, D. P. Min and M. Rho, Nucl.Phys. A585 (1995) 401

[16] M. Lutz, Phys. Lett. B426 (1998) 12

[17] A. Ramos and E. Oset, in preparation

[18] A. Pich, Rep. Prog. Phys. 58 (1995) 563

[19] G. Ecker, Prog. Part. Nucl. Phys. 35 (1995) 1

[20] V. Bernard, N. Kaiser and U. G. Meissner, Int. J. Mod. Phys. E4 (1995) 193

[21] T. S. H. Lee, J. A. Oller, E. Oset and A. Ramos, Nucl. Phys. A in print. 


\section{Figure captions}

Figure 3: Mass distribution for the different channels. The $\Sigma^{+} \pi^{-}, \Sigma^{-} \pi^{+}$ and $\Sigma^{0} \pi^{0}$ distributions are shown in the figure with the dashed lines. The solid line with the resonance shape is the sum of the three $\Sigma \pi$ channels divided by three. In addition the distributions for $\pi^{0} \Lambda$ and $K^{-} p$ production are also shown. The $\bar{K}^{0} n$ production is small and not shown there.

Figure 4: Mass distributions. Dashed line (resonant shape): $\Sigma^{0} \pi^{0}$ distribution multiplied by three. Dotted line (resonant shape): pure $\mathrm{I}=0$ contribution from the $\Sigma \pi$ channels. Solid line (resonant shape): Sum of the cross sections for all the channels. In addition the $\mathrm{I}=1$ background contribution from the $\Sigma \pi$ and $\pi^{0} \Lambda$ channels is also shown. Short-dash-dotted line: Effects of the Fermi motion with $k_{F}=268 \mathrm{MeV} / \mathrm{c}\left(\rho=\rho_{0}\right)$ where the free space $\Lambda(1405)$ distribution is assumed in the calculation. 


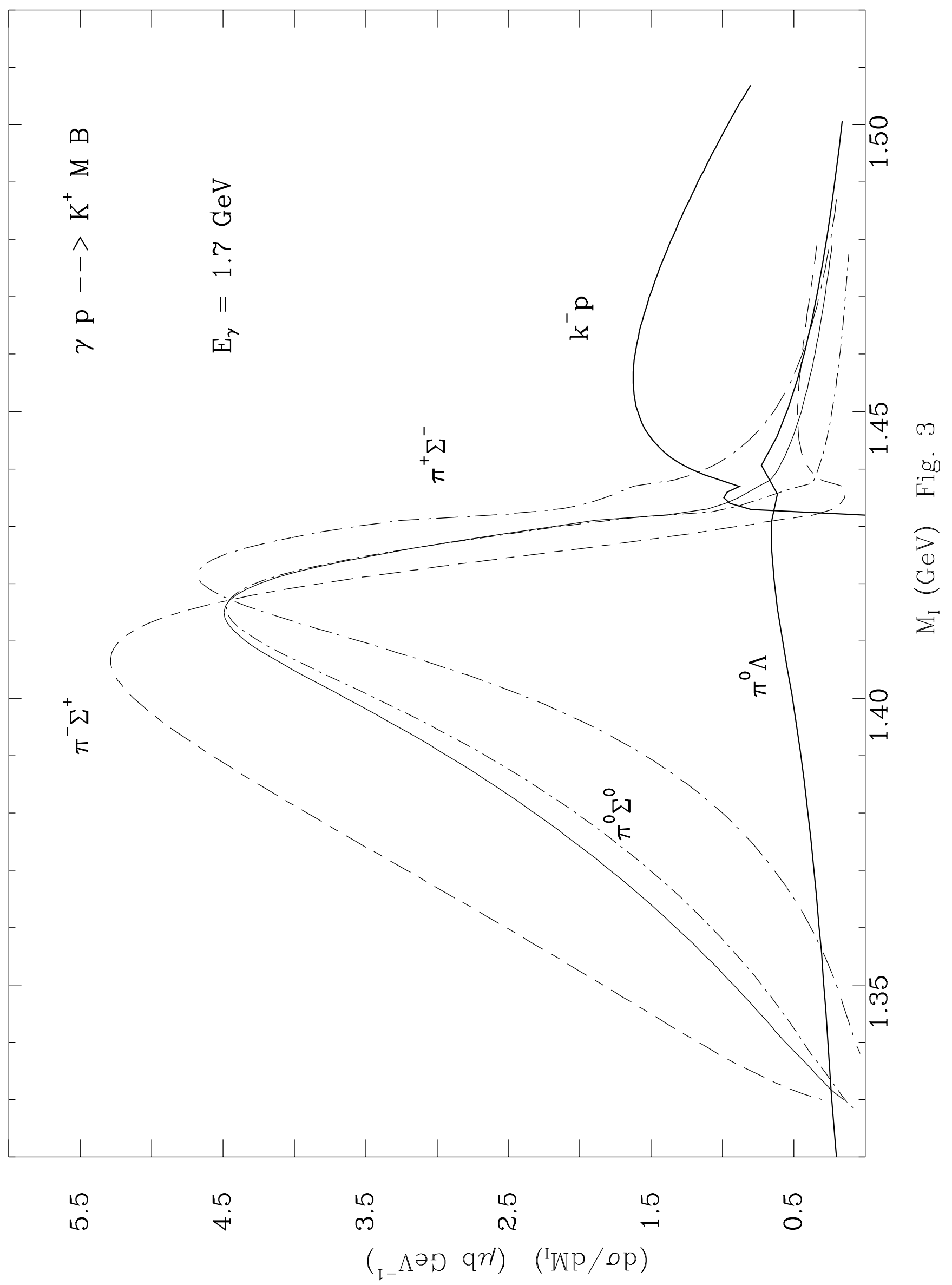




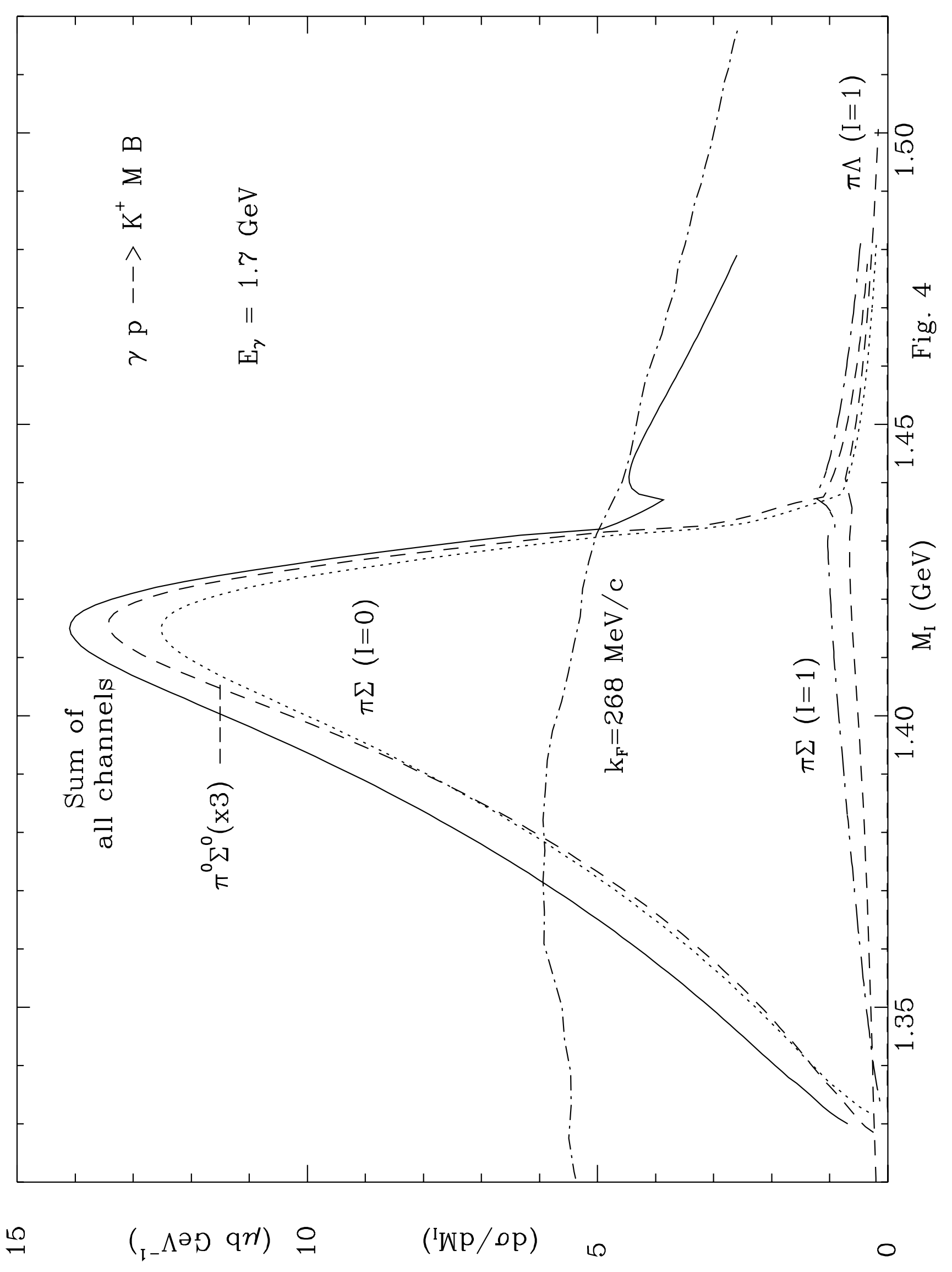

